Bull. Mater. Sci., Vol. 13, No. 3, June 1990, pp. 217-225. (C) Printed in India.

\title{
An expression for contact area between particles in a powder compact in terms of the porosity
}

\author{
N RAMAKRISHNAN* and K SIVAKUMAR \\ Defence Metallurgical Research Laboratory, Hyderabad 500 258, India
}

MS received 2 June 1989; revised 28 September 1989

\begin{abstract}
An equation for contact area between powder particles in a powder compact, in terms of the porosity, has been derived using a geometry representing spherical voids of different sizes distributed in a material matrix. This equation is verified using experimental data as well as results obtained from computer simulation of powder compaction using a finite element method.
\end{abstract}

Keywords. Contact area; porosity; spherical voids; powder compaction; finite element method.

\section{Introduction}

When an aggregate of powder particles is compacted under pressure, the point contacts between the particles flatten to areal contacts. In such a porous compact any transmission of flux such as heat, force etc takes place mainly through this contact region. Therefore any analysis involving the flow of flux invariably takes into account the contact area between the particles. Also, the contact area serves as a useful parameter in the study of sintering behaviour and the strength of the porous compact. However, the contact area cannot be determined easily by experiments and, in this context, it becomes relevant to express the contact area in terms of the porosity of the compact, which is easier to determine experimentally. In this investigation we have made an attempt to establish a relationship between contact area and porosity of the porous compact using simple geometry amenable to analytical treatment.

\section{Earlier investigations}

An experimental analysis by James (1977) brought out, rather indirectly, the variation of the contact surface area with the porosity of a powder compact. He isostatically compacted a few mono-sized metal powders and plotted the variation of a dimensionless parameter $d / d_{m}$ with the compact pressure. Here, $d$ is the diameter of the circular contact area and $d_{m}$ is the ideal limiting case of $d$, i.e. $d_{m}$ is the diameter of the circle inscribed in a pentagonal face of a regular dodecahedron of volume equal to that of the powder particle. He plotted the variation of the porosity also with the compact pressure and from these two plots it is possible to determine the variation of contact area as a function of porosity.

Fischmeister et al (1978) analysed experimentally the development of contact

\footnotetext{
*For correspondence.
} 
facets between particles during compaction of spherical bronze powder under both isostatic and uniaxial compression. He presented the variation of the contact surface area with the porosity in two plots, one describing the average number of contacts per particle, and the other representing the average contact area per contact, both as functions of porosity.

While the above two investigations were experimental in nature, Arzt (1982) attempted to establish the variation of the contact area theoretically, considering an aggregate of equi-sized spheres under isostatic compression, and each sphere filling a hypothetically constructed Voronoi polyhedron* around it. Nair and Tien (1987) determined the contact area between two spherical particles of different sizes by considering a hypothetical case where the centres of these two spheres were allowed to approach each other and the overlapping volume was smeared uniformly at the contact point in the form of a cylindrical disc. Then they extended this result to a general case considering a spatial probability distribution of these particles. Most recently Jagota and Dawson (1988) computed the variation of the contact area using finite element analysis. All these theoretical analyses first consider the variation of the average contact area per contact, later coupling it with the number of contact points per particle which also varies with densification. In this investigation we are suggesting a model which obviates the need for this intermediate step and the variation of contact area with densification is directly calculated. In fact, the equation derived in this work is much simpler than others and is reasonably supported by experimental results as well. The description of the model and its derivation are provided in the following section.

\section{The model}

Any porous body (as shown schematically in figure 1a), for the sake of computational convenience, can be approximately represented as an assembly of hollow spheres of different sizes but with each having same void fraction, which is

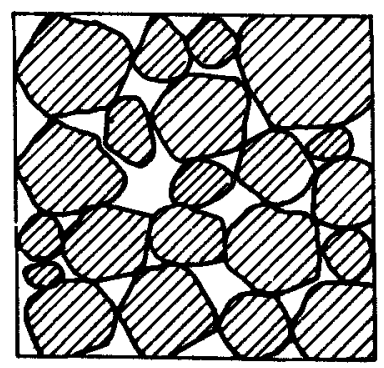

(a)

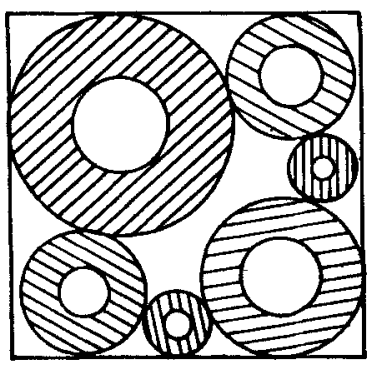

(b)

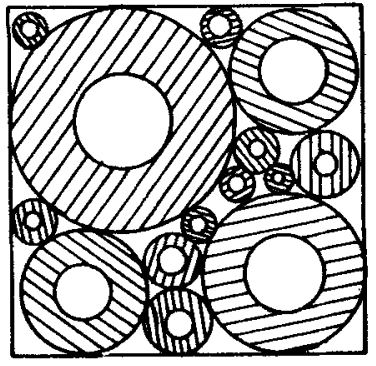

(e)

Figure 1. (a) Schematic representation of a powder aggregate. (b) and (c) Geometry of the material matrix with spherical voids of different sizes - shown as an assembly of hollow spheres with void fraction of each as same as that of the overall porosity.

\footnotetext{
*The Voronoi polyhedron of a powder particle in a packing is the set of points in space which are closer to its centre than to any other particle centre. The boundaries of this polyhedron are obtained by placing perpendicular bisecting planes on all the centre-to-centre connections. In the present context the faces of the Voronoi polyhedra contain all contact faces.
} 
equal to the overall porosity of the porous body. The logic can be explained using the following steps: First consider a box in which some hollow spheres of unequal size, but having the same void fraction, are placed as given in figure $1 \mathrm{~b}$. The remaining space can be filled up further with smaller spheres with the same porosity as given in figure 1c. This process of filling with hollow spheres can be cantinued infinitely where overall porosity of the geometry reaches the value of porosity of each individual hollow sphere. This type of arrangement essentially justifies the use of analysis of hollow spheres in real conditions.

If $b_{i}$ is the radius of the $i$ th void, the total surface area of all the spherical voids is

$$
S_{v}=4 \pi \Sigma b_{i}^{2}
$$

During densification, surface area of the voids decreases. The change in the total surface area of all the voids as a function of change in the radii can be obtained by differentiating (1), i.e.

$$
\mathrm{d} S_{v}=8 \pi \Sigma b_{i} \mathrm{~d} b_{i}
$$

and

$$
\frac{\mathrm{d} S_{v}}{S_{\mathrm{v}}}=\frac{2 \Sigma b_{i} \mathrm{~d} b_{i}}{\Sigma b_{i}^{2}}=\frac{2 \Sigma b_{i}^{2}\left(\mathrm{~d} b_{i} / b_{i}\right)}{\Sigma b_{i}^{2}}
$$

Here, $\mathrm{d} b_{i} / b_{i}$ being independent of ' $i$ (rigorous proof is supplied in the appendix), it can be taken out as a factor and (3) reduces to

$$
\frac{\mathrm{d} S_{v}}{S_{v}}=\frac{2 \mathrm{~d} b_{i}}{b_{i}}
$$

The total porosity or void fraction $\theta$ can be written as

$$
\theta=\frac{(4 / 3) \pi \Sigma b_{i}^{3}}{V_{m}+(4 / 3) \pi \Sigma b_{i}^{3}}
$$

The change in $\theta$ with $b_{i}$ can be obtained by differentiating (5),

$$
\mathrm{d} \theta=\frac{(1-\theta) 4 \pi \Sigma b_{i}^{2} \mathrm{~d} b_{i}}{V_{m}+(4 / 3) \pi \Sigma b_{i}^{3}}
$$

combining (5) and (6) we get

$$
\frac{\mathrm{d} \theta}{\theta(1-\theta)}=\frac{3 \Sigma b_{i}^{2} \mathrm{~d} b_{i}}{\Sigma b_{i}^{3}}=\frac{3 \Sigma b_{i}^{3}\left(\mathrm{~d} b_{i} / b_{i}\right)}{\Sigma b_{i}^{3}}
$$

$\mathrm{d} b_{i} / b_{i}$ being independent if $i$ (as given in the appendix)

$$
\frac{\mathrm{d} \theta}{\theta(1-\theta)}=\frac{3 \mathrm{~d} b_{i}}{b_{i}} \text {. }
$$

Then, (4) and (8) can be combined to give

$$
\frac{\mathrm{d} S_{v}}{S_{v}}=(2 / 3) \frac{\mathrm{d} \theta}{\theta(1-\theta)}
$$

The solution of this differential equation is

$$
\ln \left(S_{v} / S_{0}\right)=(2 / 3)\left\{\ln [\theta /(1-\theta)]-\ln \left[\theta_{0} /\left(1-\theta_{0}\right)\right]\right\}
$$


It must be clear from figure 1a that the sum of the total contact area between all the particles and the total surface area of all the voids must be equal to the total surface area of all the particles at any given instant of densification. For the sake of convenience if we choose $\left(1-\theta_{0}\right)$ to be the tap density itself, in which case the particles are just in contact with each other without the development of any contact area. $S_{0}$ becomes the total surface area of all the particles. Also, as the total surface area of the particles does not vary significantly with densification, we can slightly modify (10) and write it as

$$
\ln \left[1-\left(S_{c} / S_{0}\right)\right]=(2 / 3)\left\{\ln [\theta /(1-\theta)]-\ln \left[\theta_{0} /\left(1-\theta_{0}\right)\right]\right\} .
$$

\section{Experimental verification}

In the previous section we assumed the voids to be spherical to facilitate analytical treatment. But in a real case the voids are of complex shape and it may not be reasonable to directly apply the equation derived for such conditions. Nevertheless it can be supposed that the general nature of the expression relating the contact area and the porosity remains independent of the shape of the voids. Then (11) can be written in a semi-empirical fashion to suit real cases, i.e.

$$
\ln \left[1-\left(S_{c} / S_{0}\right)\right]=m \ln [(\theta / 1-\theta)]+C .
$$

For spherical voids, $m=2 / 3$ and $C=-(2 / 3) \ln \left[\theta_{0} /\left(1-\theta_{0}\right)\right]$. We took the experimental data from James (1977) and Fischmeister et al (1978) and plotted $\ln [\theta /(1-\theta)]$ vs $\ln \left[1-\left(S_{c} / S_{0}\right)\right]$ as shown in figures 2 and 3 respectively. For the data

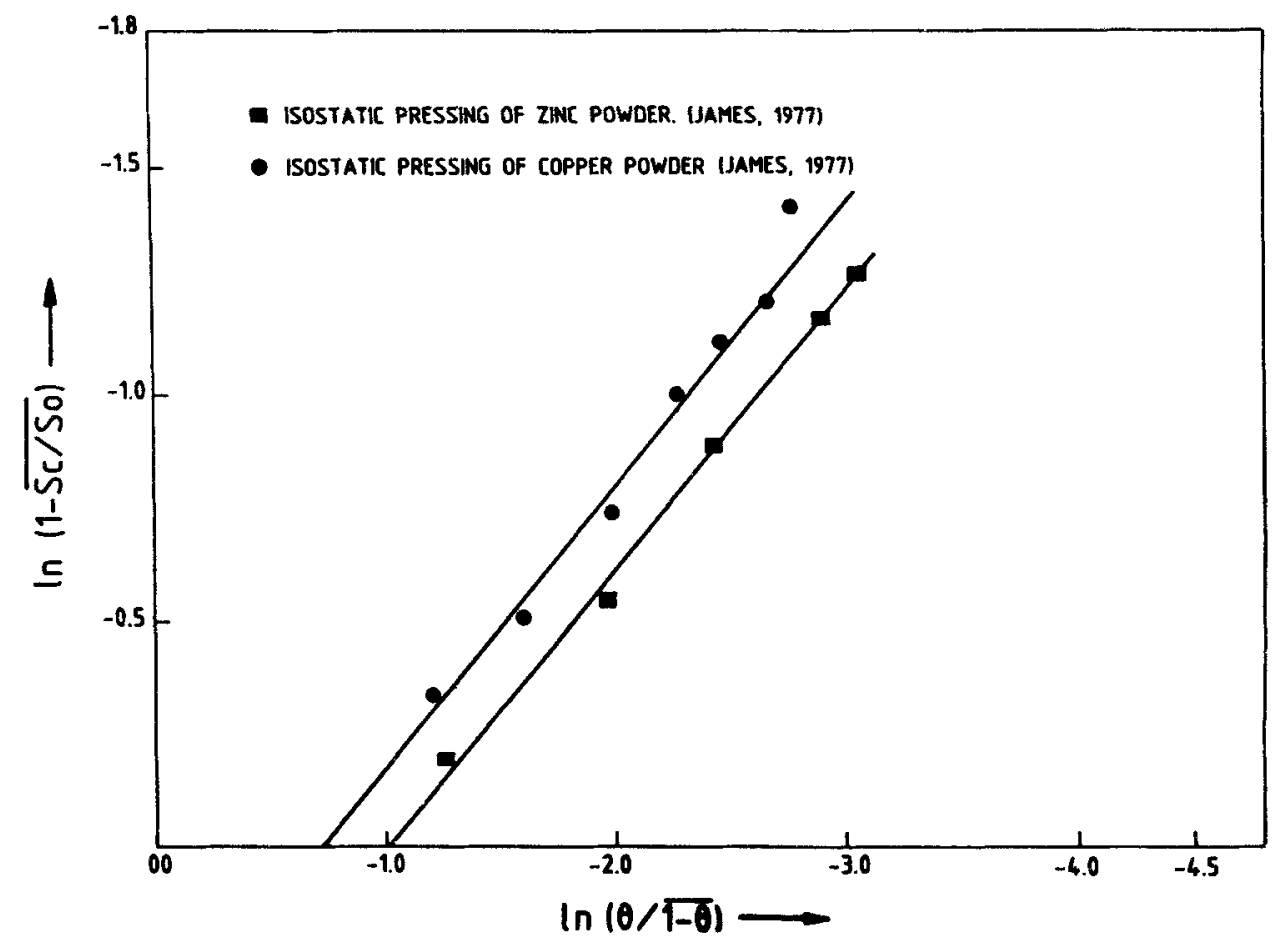

Figure 2. Experimental verification of (12) with the data of James (1977). 


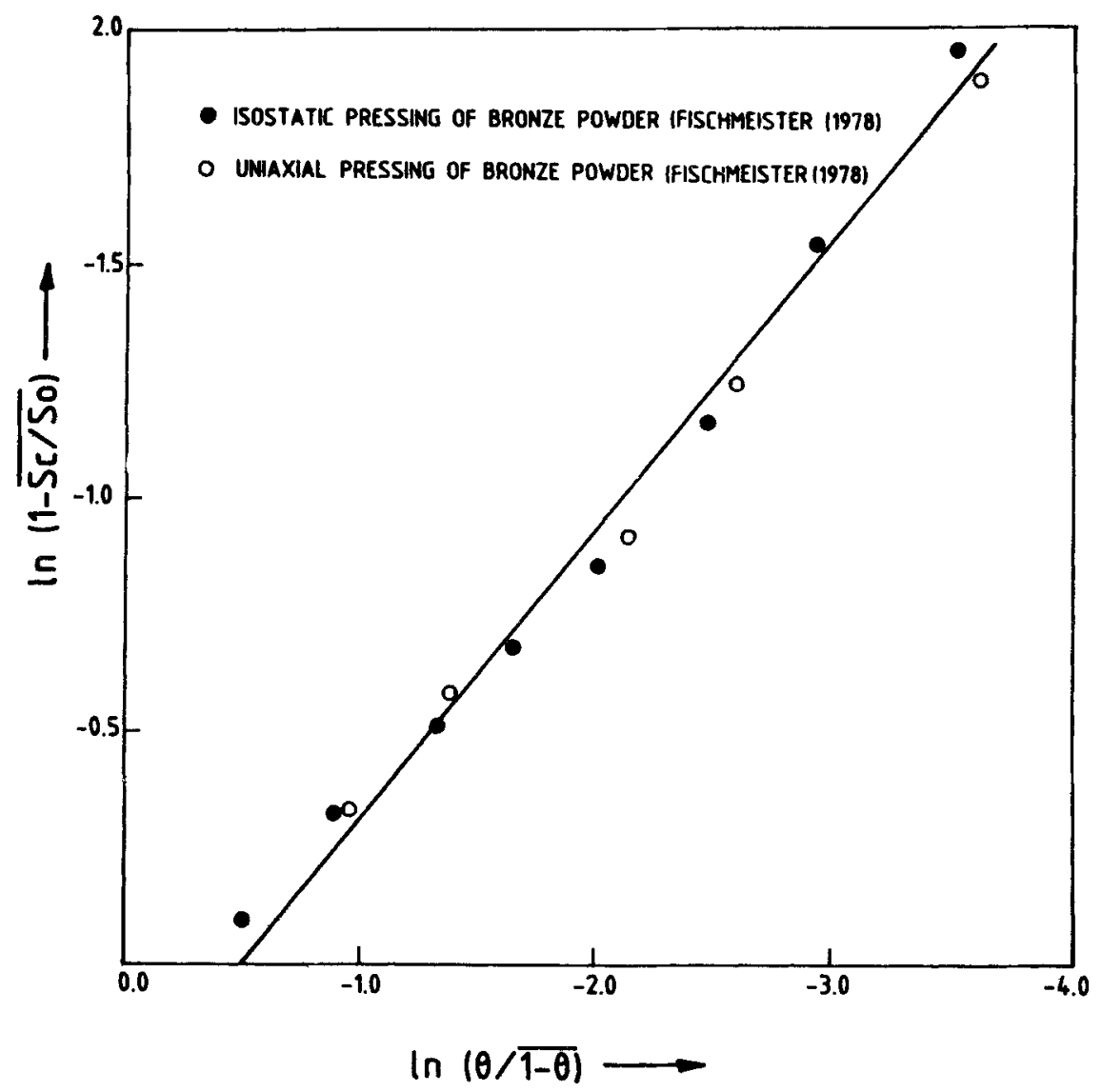

Figure 3. Experimental verification of (12) with the data of Fischmeister et al (1978).

corresponding to both atomised copper and zinc powders (James 1977), the value of $m$ turned out to be $0 \cdot 67$; but the value of $C$ differed for copper and zinc and was found to be 0.542 and 0.678 respectively. In the case of atomised bronze powder (Fischmeister et al 1978), $m$ was found to be 0.60 and $C$ to be 0.28 for both isostatic and uniaxial compaction.

Apart from the above, we have also conducted computer simulation studies using the finite element method. A geometry as shown in figure 4 , representing a powder aggregate, was chosen for this purpose. The details about the finite element algorithm, the geometry considered and the procedure for imposing appropriate boundary conditions can be found elsewhere (Ramakrishnan 1988; Ramakrishnan et al 1984). We analysed the following 3 cases in this investigation:

(a) isostatic pressing of nickel powder,

(b) uniaxial pressing of nickel powder,

(c) isostatic pressing of aluminium powder.

To computer simulate the above, the data describing the stress-strain relationship of both nickel and aluminium were fed as inputs and suitable boundary conditions were imposed under two-dimensional plane-strain conditions. The pressure was 
(a)



POROSITY $=0.1309$

(c)

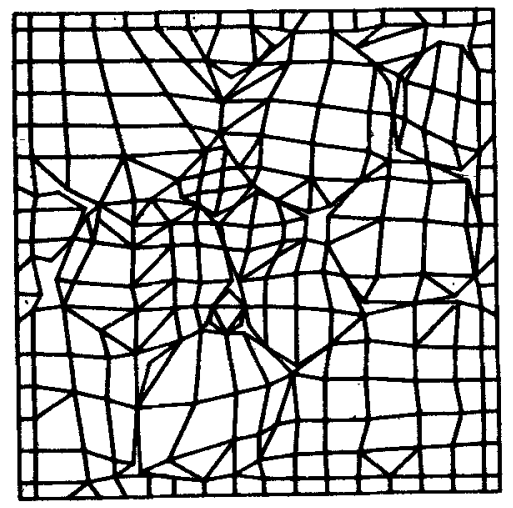

POROSITY $=0.0356$ (b)

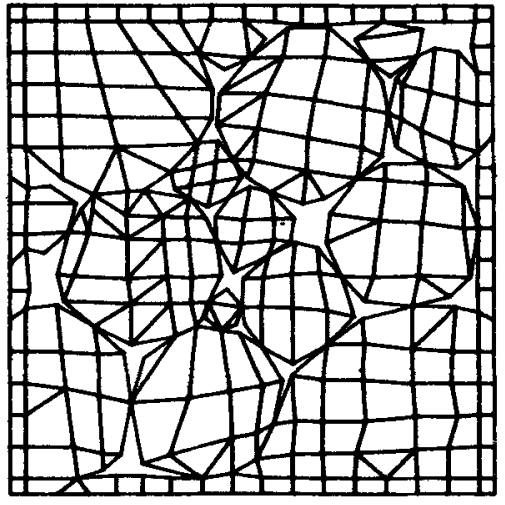

POROSITY $=0.0748$

(d)



POROSITY $=0.0182$

Figure 4. Computer simulation of powder compaction using the finite element method.

increased in small steps and the geometry at the end of each step was recorded. Out of these geometries four selected cases are shown in figure 3. The total surface area of all the particles (cireular discs in this case) and the contact area between all the particles, at each porosity, were measured physically. Using this data, plots between $\ln [\theta /(1-\theta)]$ and $\ln \left[1-\left(S_{c} / S_{0}\right)\right]$ were drawn for the three cases mentioned above. The values of $m$ and $C$ for all these cases were found to be 0.4347 and 1.03 respectively. The theoretical value of $m$ for a geometry with circular holes is $1 / 2$ which can be derived using the same procedure outlined in $\$ 3$. Therefore to adapt the $m$ value obtained in a two-dimensional plane-strain condition to the threedimensional case, a factor of $4 / 3$ has to be used and with this factor, the value of $m$ works out to be 0.579 . With the factor $4 / 3$ the value of $C$ becomes $1 \cdot 37$. Here again, we find $m$ and $C$ to be independent of the type of pressing and interestingly, it appears to be independent of material as well if the shape of the powder particles is the same. 


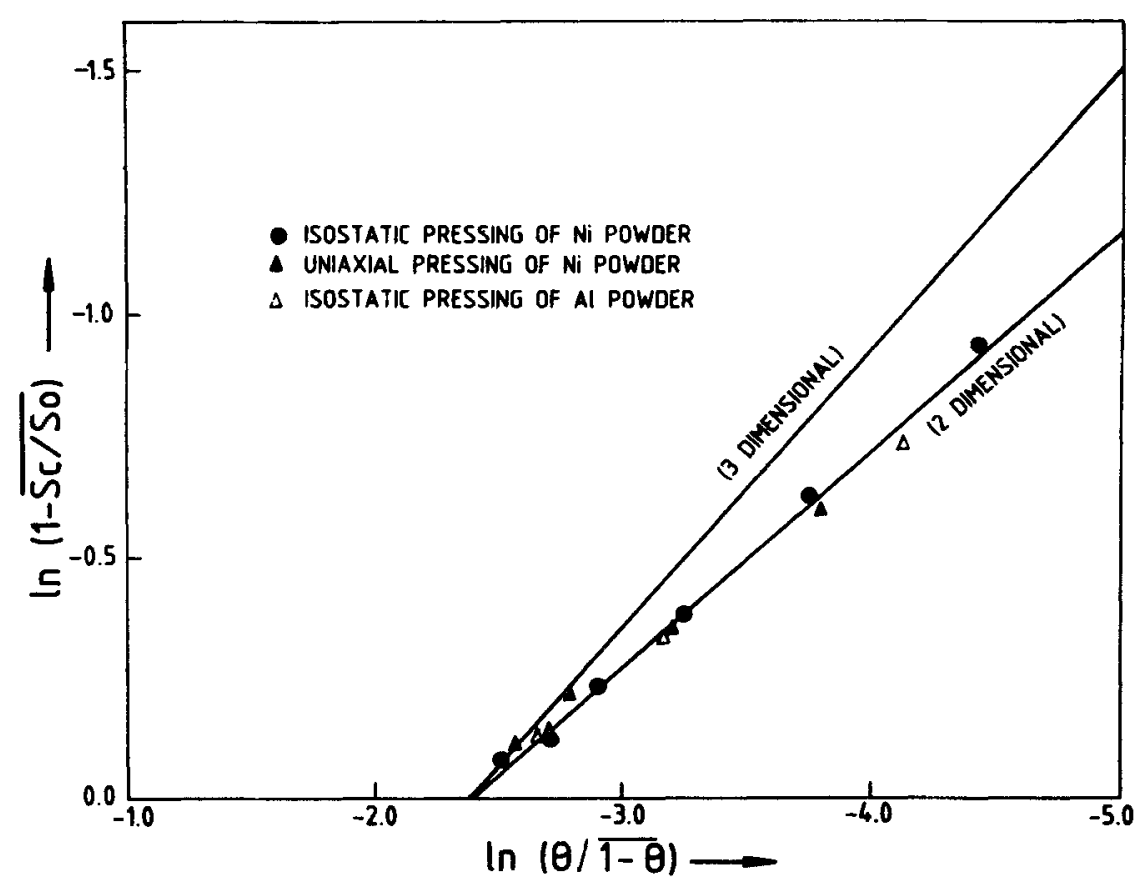

Figure 5. Verification of (12) with the data obtained using computer simulation.

\section{Summary}

This investigation essentially brings out the variation of contact area between powder particles in a powder aggregate when it undergoes compaction. Though a few workers have dealt with this problem in the past both experimentally and theoretically in different contexts, a simple and convenient equation for expressing the contact area as a function of the porosity was not available so far. In this work we have selected the geometry of the material matrix with spherical voids and derived the relationship rigorously and later extended it to a general case in a semiempirical fashion. The relationship can be expressed in simple form as,

$$
\left(S_{c} / S_{0}\right) \propto 1-[\theta /(1-\theta)]^{m}
$$

where $S_{c} / S_{0}$ is normalised contact area between the powder particles and $m$ is a constant approximately equal to $2 / 3$. The published data and the data from the computer simulation studies agree with this equation very well confirming its validity under different conditions.

\section{Appendix}

Figure 1c depicts how a material matrix with spherical voids of different sizes is constructed as an assembly of fictitious hollow spheres each with a void fraction equal to the porosity of the entire geometry. Consider an arbitrarily chosen hollow sphere from figure 1c. When this sphere shrinks uniformly towards the centre, the 
strain distribution in the sphere satisfies the following conditions:

$$
\begin{aligned}
& \frac{\partial\left(r \varepsilon_{t}\right)}{\partial r}=\varepsilon_{r}, \text { (compatibility condition) } \\
& \varepsilon_{r}=2 \varepsilon_{t}, \text { (volume constancy condition) }
\end{aligned}
$$

where $\varepsilon_{r}$ and $\varepsilon_{t}$ are the radial and tangential strains at a distance $r$ from the centre of the sphere. The radial strain is

$$
\varepsilon_{r}=\frac{\partial u}{\partial r}
$$

where $u$ is the displacement at a distance $r$ from the centre. Combining (A1) to (A3) we can show that

$$
-(1 / 2) \varepsilon_{r}=u / r
$$

Therefore at $r=a$,

$$
-(1 / 2) \varepsilon_{a}=\Delta a / a
$$

where $\Delta a$ is the displacement at $r=a$. As the volume of the material of the sphere remains constant

$$
b^{2} \Delta b=a^{2} \Delta a,
$$

where $\Delta b$ is the displacement at $r=b$. Combining (A5) and (A6) we get,

$$
\Delta b / b=(1 / \theta) \Delta a / a=-(1 / 2)\left(\varepsilon_{a} / \theta\right),
$$

where $\theta$ is the void fraction equal to $b^{3} / a^{3}$ which is independent of the hollow sphere chosen. The strain at the outer surface of any arbitrarily chosen hollow sphere should be equal to the strain at the outer surfaces of all the hollow spheres in contact with it. This condition is essential to satisfy the compatibility between the hollow spheres, in other words, the continuity in the strain distribution in the material matrix. Therefore the strain at the outer surface of all the spheres should be equal, i.e. $\varepsilon_{a}$ is also independent of the hollow sphere chosen. With this and using (A7) it can be shown that $\Delta b / b$ is also independent of the hollow sphere chosen.

\section{List of symbols}

$a_{i}, b_{i} \quad$ outer and inner diameters of the $i$ th hollow sphere in figure $1 \mathrm{c}$, respectively;

$\Delta a, \Delta b$ displacement at the outer and inner surfaces or change in the outer diameter $a$ or the inner diameter $b$, respectively, of the hollow sphere chosen;

$C y$-intercept of the linear plot between $\ln [\theta /(1-\theta)]$ and $\ln \left[1-\left(S_{c} / S_{0}\right)\right]$;

$m \quad$ slope of the linear plot between $\ln [\theta /(1-\theta)]$ and $\ln \left[1-\left(S_{c} / S_{0}\right)\right]$;

$r \quad$ radial distance, as a subscript it means 'radial';

$S_{c} \quad$ total contact area between all the particles;

$S_{0} \quad$ total surface area of all the particles or total surface area of all the voids when $\theta=\theta_{0}$;

$S_{v} \quad$ total surface area of all the voids; 
$u \quad$ displacement;

$V_{m} \quad$ volume of the material portion of the powder compact;

$\varepsilon_{r}, \varepsilon_{t} \quad$ radial and tangential strains, respectively;

$\varepsilon_{a} \quad$ radial strain at the outer surface of the hollow sphere;

$\theta$ porosity;

$\theta_{0} \quad$ initial porosity or ' 1 - tap density';

$\Sigma \quad$ summation from 1 to $n$ number of voids.

\section{References}

Arzt E 1982 Acta Metall. 301883

Fischmeister H F, Arzt E and Olsson L R 1978 Powder Metall. 21179

Jagota A and Dawson P R 1988 Acta Metall. 362551

James P J 1977 Powder Metall. 20199

Nair S V and Tien J K 1987 Metall. Trans. A18 97

Ramakrishnan $\mathrm{N} 1988$ An analysis of powder consolidation by computer simulation using finite element method, Ph. D. thesis, Banaras Hindu University, Varanasi.

Ramakrishnan N, Balakrishna Bhat T and Arunachalam V S 1984 Acta Metall. 32357 